\title{
Caractérisation des faibles niveaux de radioactivité d'échantillons végétaux par étude de la décroissance. Appli- cation au double marquage ${ }^{32} \mathrm{P} /{ }^{35} \mathrm{~S}$
}

\author{
Robert HABIB, Franck ARIES(*) \& Roger GUENNELON(**). \\ I.N.R.A., Station d'Agronomie, \\ (*) Station de Biométrie, \\ (**) Station de Science du Sol, Centre de Recherches d'Avignon, BP 91, F 84140 Montfavet.
} actuelle.

Mots clés additionnels : Traceur radioactif, pommier, statistique comptage, ${ }^{40} \mathrm{~K}$, bruit de fond.

Characterization of low radioactive levels in plant samples by the study of count decrease. Application to ${ }^{32} P /{ }^{35} S$ double labelling.

The authors propose a method for dealing with low levels of radioactive contamination of plant tissues and high background variability. It is based on statistical analysis of radioactive decrease models. This method allows the use of double labelling and is very simple for single labelling. Only simple equipment for measuring and computing is needed.

Additional key-words : Radioactive tracer, apple, statistics of counts, ${ }^{40} \mathrm{~K}$, background.

\section{INTRODUCTION}

Les expériences sur plantes pérennes au champ utilisant des traceurs radioactifs se heurtent à une difficulté certaine : la nécessité de ne pas utiliser de fortes activités de radioéléments, le volume important du sol exploré par les racines, la grande masse de produit végétal (tronc, branches, feuilles, etc.), tous ces facteurs conduisant à de faibles taux de radioactivité dans les prélèvements que l'on est amené à faire. Au cours d'un travail précédemment réalisé (HABIB), 1981), on a même volontairement limité l'apport de traceur radioactif (en l'occurrence ${ }^{32} \mathrm{P}$ et ${ }^{35} \mathrm{~S}$ ) à un petit volume de sol, de l'ordre de quelques $\mathrm{cm}^{3}$. Le problème posé était alors de savoir s'il y avait eu ou non absorption de ces éléments, se traduisant par une contamination du feuillage. Pour les raisons évoquées ci-dessus, les échantillons de feuilles était donc très faiblement marqués.

La contamination des feuilles prélevées sur chaque arbre traité devait donc être appréciée quant à son origine ${ }^{32} \mathrm{P}$ et/ou ${ }^{35} \mathrm{~S}$ ), et ceci par rapport à un bruit de fond non négligeable de radioactivité naturelle, s'ajoutant au mouvement propre de l'appareil de comptage (ensemble environnement, appareil, support des échantillons).

Ces expériences ne nécessitent pas obligatoirement d'accéder à la véritable valeur de l'activité de l'échantillon. En effet, on veut essentiellement, parmi une population d'arbres traités de façon identique, déterminer ceux qui, par suite des conditions physiques du point de contamination dans le sol, ont (ou n'ont pas) absorbé les éléments injectés au niveau des racines.

La certitude de la réponse dépendra du coefficient de variation de la valeur du rapport signal sur bruit de fond. Il est donc nécessaire que les 2 termes de ce rapport soient connus avec la plus grande précision possible. Ceci implique soit de disposer de méthodes de comptage très sensibles, soit de procéder à des comptages longs.

Le problème de bruit de fond n'est pas cependant résolu pour autant, car la variabilité de la teneur ${ }^{40} \mathrm{~K}$, souvent principal responsable de la radioactivité naturelle des échantilons végétaux, est importante. De plus, lorsqu'on utilise, comme c'est le cas dans nos expériences, un double marquage, il faudrait, lors de comptages longs, corriger les 
mesures en fonction des périodes des éléments considérés, opération impossible puisqu'on ne connaît pas leurs proportions relatives dans l'échantillon.

Nous avons cherché à utiliser une méthodologie de comptage qui nous dispense, théoriquement, de connaître, $a$ priori, le bruit de fond global du système échantillonappareil de mesure.

On a donc proposé une technique basée sur l'ajustement de la courbe expérimentale de décroissance du taux de comptage de l'échantillon, en fonction du temps, à un modèle paramétré par les périodes de 2 radioéléments. Elle a été exposée, dans son principe, pour le cas de la spectrométrie Gamma (QUITTNER, 1973). Cette méthode s'applique à des taux de comptage différents du bruit de fond, car elle permet d'obtenir une bonne estimation de ce paramètre, pour l'échantillon considéré. Cette estimation découle de l'ajustement du modèle aux valeurs expérimentales.

Elle est applicable à des mélanges d'isotopes radioactifs à condition que les périodes soient suffisamment différentes, faute de quoi l'allure des courbes de décroissance globale serait presque invariante avec les proportions relatives des radioisotopes.

Les mesures peuvent être faites, sur des échantillons végétaux séchés et broyés, à l'aide d'un compteur à flux gazeux. On notera toutefois que le but de la mise en œuvre de cette méthode est d'aboutir à une réponse de type logique : contamination ou absence de contamination. Les améliorations de la statistique du comptage n'auront pour effet que de diminuer le nombre de cas où la réponse est incertaine. La signification globale du résultat recherché peut donc l'être en optimisant les mesures de radioactivité ou en augmentant le nombre d'individus traités. Selon les impératifs d'équipement, de temps ou de disponibilité, de matériel et de personnel, l'une ou l'autre des démarches peut être adoptée.

La méthode proposée offre l'avantage de la simplicité et a, dans le cas de l'expérience relatée par ailleurs (HABIB, 1981), permis d'obtenir, avec la valeur du niveau de signification des résultats, la réponse à un problème agronomique posé.

Cette méthode, si elle peut paraitre rudimentaire sur le plan métrologique eu égard à la technologie actuelle des mesures de faible niveau de contamination (IAEA, 1967, 1981), peut être utilisée dans les laboratoires possédant un équipement simple quant aux moyens de mesure et de calcul ; laboratoires dont la localisation géographique peut rendre difficile la maintenance du matériel utilisé.

\section{MATÉRIEL ET MÉTHODES}

\section{A. Dispositif expérimental de contamination}

Nous avons employé 2 radioéléments :

- ${ }^{32} \mathrm{P}$ de période $14,3 \mathrm{j}$.,

- ${ }^{35} \mathrm{~S}$ de période $87 \mathrm{j}$.

dont nous avons testé l'absorption par le système racinaire de pommier (Golden delicious/M2). L'ensemble du travail a été effectué sur un verger de 36 arbres plantés en 1975, en 4 rangs notés de I à IV (espacement $4 \times 4,5 \mathrm{~m}$ ). Le rang I est bordé par une haie de cyprès et le rang IV par le che- min d'accès à la parcelle. Le sol est un limon argilo-calcaire (limon de St-Paul).

L'essai a eu lieu au mois d'août 1979.

La solution radioactive $1,55 \times 10^{7} \mathrm{~Bq}$ de ${ }^{32} \mathrm{P}$ et de 2,07 $\times 10^{7} \mathrm{~Bq}$ de ${ }^{35} \mathrm{~S}$ en solution acide, complétée à $5 \mathrm{~cm}^{3}$ par de l'eau distillée, a été injectée dans le sol, sans entraîneur, à $35 \mathrm{~cm}$ de profondeur, à raison d'un point d'injection par arbre. Les coordonnées de ce point d'injection sont tirées au hasard à l'intérieur de la surface irriguée. Puis l'on a rincé avec 2 fois $5 \mathrm{~cm}^{3}$ d'eau distillée. La justification de ce protocole a été exposée par ailleurs (HABIB, 1981).

\section{B. Mesures de radioactivité}

L'échantillonnage des parties aériennes, pour y rechercher la présence de l'un et (ou) l'autre des 2 radioéléments, a été réalisé par le prélèvement au hasard de 20 feuilles dans le tiers inférieur de couronne de l'arbre, et ce, $2 \mathrm{~h}$ après l'injection de la solution radioactive. Ce délai était volontairement court pour permettre de considérer les conditions physiques du sol comme homogènes et constantes pendant la phase d'absorption racinaire. Les prélèvements des 36 échantillons (1 échantillon par arbre) se sont échelonnés entre $10 \mathrm{~h}$ et $16 \mathrm{~h} 30$.

Les feuilles ont été séchées dans une étuve à $70^{\circ} \mathrm{C}$ et broyées finement ; $1 \mathrm{~g}$ de poudre végétale a été déposé dans une coupelle en acier inoxydable de $8 \mathrm{~cm}$ de diamètre et étalé en couche mince après avoir été mis en suspension dans une solution d'alcool contenant 40 p. 100 de Tween. Ceci permet d'obtenir des couches pouvant être considérées comme homogènes. En outre, la pellicule pâteuse obtenue permet, après séchage, de manipuler et de conserver les coupelles sans perte d'échantillon.

Bien que l'on n'ait pas recherché une interprétation quantitative des estimations de taux de comptage, il faut noter que la reproductibilité du remplissage des coupelles aurait pu permettre une mesure quantitative grâce à un étalonnage au ${ }^{32} \mathrm{P}$ et au ${ }^{35} \mathrm{~S}$, pour corriger les interactions avec la matrice.

On a mesuré le taux de comptage radioactif des échantillons de feuilles dans un compteur proportionnel à flux gazeux, à bas bruit de fond. Pour des compteurs de diamètre utile de $135 \mathrm{~mm}$, le constructeur indique un mouvement propre de 6 à 8 impulsions par mn pour une géométrie de garde de $4 \pi$ (soit 300 à 480 impulsions par h, iph, appareil vide).

Pour un même échantillon, on a effectué 10 à 11 comptages, à des temps variant de 0 à $130 \mathrm{j}$ depuis le temps $\mathrm{t}_{\mathrm{s}}$ $=0 \mathrm{du} 1^{\text {er }}$ comptage. Les 36 échantillons ont toujours été comptés les uns après les autres dans le même ordre; il y a donc au début de l'essai $36 \mathrm{~h}$ de décalage entre le $1^{\text {er }}$ et le dernier échantillon mesuré. Chaque série de 36 comptages a été précédée de la mesure d'un étalon ${ }^{90} \mathrm{Sr}$. Ceci nous permet de contrôler l'amplitude des modifications éventuelles de rendement de comptage au cours de l'essai.

Les activités de toutes les coupelles vides et supports de coupelles ont été mesurées plusieurs jours avant le comptage des échantillons et tout le matériel dont la mesure a excédé $10 \mathrm{ipm}$ a été ou nettoyé et retesté ou écarté (mouvement propre de l'installation : 5 à $8 \mathrm{ipm}$ ).

La durée de comptage de chaque échantillon était de $1 \mathrm{~h}$ pour les 7 ou 8 premières séquences de comptage, puis de $4 \mathrm{~h}$ pour les séquences suivantes, c'est-à-dire environ $75 \mathrm{j}$ après le début des mesures. 


\section{Méthode d'ajustement des courbes de décroissance}

\section{Principe général}

Dans le cas d'un double marquage, on peut considérer que le taux de comptage mesuré d'un échantillon végétal a 4 origines :

- le $1^{\text {er }}$ isotope utilisé de période $T_{1}$,

- le $2^{e}$ isotope de période $T_{2}$,

- le bruit de fond lié à la radioactivité naturelle de l'échantillon : essentiellement ${ }^{40} \mathrm{~K}$, en l'absence de contamination accidentelle,

- le bruit de fond propre de l'enceinte de mesure (coupelle et support de coupelle compris).

Les taux de comptage dus aux 2 dernières composantes de la radioactivité peuvent être sommés, si l'on considère la période du ${ }^{40} \mathrm{~K}\left(1,3 \times 10^{9}\right.$ années) comme infinie par rapport à notre échelle de temps.

A l'origine du temps, $t_{1}$, que l'on peut prendre au moment du $1^{\text {er }}$ comptage effectué pour l'échantillon considéré, on peut alors décomposer la radioactivité totale en ses 3 composantes :

- $c_{1}$ radioactivité due au $1^{\text {er }}$ isotope,

- $c_{2}$ radioactivité due au $2^{\mathrm{e}}$ isotope,

- $\mathrm{c}_{\mathrm{B}}$ bruit de fond global,

$c=c_{1}+c_{2}+c_{B} \quad\left(t_{1}=0\right)$

Plus généralement, et compte tenu de la décroissance de chaque radioélément de périodes $T_{1} T_{2}$, on dispose du modèle :

$c(t)=c_{1} \exp \left(-0,693 \frac{t}{T_{1}}\right)+c_{2} \exp \left(-0,693 \frac{t}{T_{2}}+c_{B}\right)(1)$

où $\mathrm{c}(\mathrm{t})$ représente la radioactivité totale au temps $\mathrm{t}$.

Le problème revient à estimer les paramètres $c_{1}, c_{2}$ et $c_{B}$.

Pour faire cette estimation, on dispose, pour chaque échantillon, de $\mathrm{n}$ comptages effectués à différents temps $\mathrm{C}\left(\mathrm{t}_{1}\right), \mathrm{C}\left(\mathrm{t}_{2}\right), \ldots \mathrm{C}\left(\mathrm{t}_{\mathrm{n}}\right)$ qui permettront le calcul des valeurs $\hat{c}_{1}, \quad \hat{c}_{2}, \quad \hat{c}_{\mathrm{B}}$ s'ajustant «au mieux » aux données expérimentales.

Pour résoudre ce problème, on peut faire le changement de variable :

$$
\begin{aligned}
& x_{1 i}=\exp \left(-0,693 \frac{t_{i}}{T_{1}}\right) \\
& x_{2 i}=\exp \left(-0,693 \frac{t_{i}}{T_{2}}\right) \quad i=1, \ldots, n
\end{aligned}
$$

Les 2 nouvelles variables sont liées par la relation : $x_{1 i}$ $=\mathrm{x}_{2 \mathrm{i}} \mathrm{T}_{2} / \mathrm{T}_{1}$ ce qui explique la corrélation signalée plus loin.

Le modèle devient :

$c\left(t_{i}\right)=c_{1} x_{1 i}+c_{2} x_{2 i}+c_{B}$

L'estimation de $c_{1}, c_{2}$ et $c_{B}$ pourrait alors se faire par la méthode des moindres carrés en minimisant la quantité résiduelle $\mathrm{S}$, somme des carrés des écarts au modèle, soit en écriture matricielle :

$S=\left(C_{t}-X . \hat{c}\right)^{\prime}\left(C_{t}-X . \hat{c}\right)$

$C_{t}$ est la matrice des taux de comptage $C\left(t_{i}\right)$

[n.1]

$X$ est la matrice des $x_{k i} k=1, \ldots, p$

$[\mathrm{n} .(\mathrm{p}+1)]$

$\hat{c}$ est la matrice des estimateurs

$[(p+1) .1]$

Le symbole «'" est utilisé pour indiquer la transposée de la matrice considérée, qui est obtenue en échangeant les lignes et les colonnes; on a indiqué entre crochets les dimensions des matrices, $\mathrm{p}$ étant le nombre de radioéléments utilisés.
Si on veut tester les paramètres du modèle linéaire, 2 conditions doivent être remplies :

$1^{\circ}$ Les taux de comptage $C\left(t_{1}\right)$ doivent être indépendants et de même variance.

$2^{\circ}$ La distribution des erreurs $\epsilon_{i}$ doit être normale et d'espérance nulle.

Or, si $c(t)$ est la radioactivité au temps $t, C(t)$, mesure du taux de comptage, suit une loi de POISSON. Compte tenu de l'ordre de grandeur du paramètre (plusieurs centaines d'impulsions), on peut l'approcher par la loi normale.

On peut admettre que la condition de normalité sera respectée, au moins pour la partie de l'erreur liée au caractère aléatoire de l'émission radioactive.

\begin{tabular}{|c|c|}
\hline $\begin{array}{l}\mathrm{C}\left(\mathrm{t}_{\mathrm{i}}\right) \\
\text { omptage } \\
\text { mesuré }\end{array}$ & $\begin{array}{c}\hat{\mathrm{c}}\left(\mathrm{t}_{\mathrm{i}}\right) \\
\text { radioactivité } \\
\text { estimée }\end{array}$ \\
\hline
\end{tabular}

On peut alors écrire le modèle statistique :

où $\epsilon_{\mathrm{i}}$ suit une loi normale, de moyenne nulle et de variance $\mathrm{C}\left(\mathrm{t}_{\mathrm{i}}\right)$ (on remplace au niveau de la variance la valeur inconnue $c\left(t_{i}\right)$ par son estimation $\left.\hat{C}\left(t_{j}\right)\right)$.

Par contre, la condition d'égalité des variances n'est pas observée puisque, lors de la décroissance des comptages, la variance décroît conjointement. En effet, comme nous venons de le voir, on a fait l'approximation :

$\operatorname{Var} C\left(t_{i}\right)=C\left(t_{i}\right)$

Ainsi, pour se rapprocher des spécifications du modèle linéaire qui est utilisé, il est nécessaire d'effectuer une régression pondérée :

Si D est la matrice diagonale des poids, $w_{i i}$, la méthode des moindres carrés conduira à minimiser $\mathrm{S}_{\mathrm{p}}$, la somme des carrés des écarts au modèle, pondérés par l'inverse des poids associés à chaque mesure :

$S_{p}=\left(C_{t}-X . \hat{c}\right)^{\prime} D^{-1}\left(C_{t}-X . \hat{c}\right)$

$S_{p}=\left(C_{t}^{\prime} \cdot D^{-1 / 2}-\hat{c} \cdot X^{\prime} \cdot D^{-1 / 2}\right)\left(D^{1 / 2} \cdot C_{t}-D^{-1 / 2} \cdot X \cdot c\right)(5)$

Si on pose $w_{i i}=\operatorname{Var} C\left(t_{j}\right)$, les $C\left(t_{i}\right)$ pondérés, soit $\mathrm{C}\left(\mathrm{t}_{\mathrm{i}}\right)^{1 / 2}$, suivent la condition d'égalité des variances.

L'équation (4), développée en (5), montre qu'il est nécessaire d'effectuer une régression sans terme constant ; car la matrice $\mathrm{D}^{-1 / 2} \mathrm{X}$ inclut les constances pondérées 1.C $\left(\mathrm{t}_{\mathrm{j}}\right)^{1 / 2}$, qui jouent alors le rôle d'une variable.

La minimisation de $S_{p}$ se ramène donc au problème classique de la régression multiple sans terme constant. Les 3 coefficients $\hat{c}_{1}, \hat{c}_{2}$ et $\hat{c}_{\mathrm{B}}$ pourront être obtenus par un programme standard REPRG. (BACHACOU et al., 1981).

\section{Utilisation de mesures ayant des temps de comptage différents}

Nous avons supposé, jusqu'à présent, que tous les comptages étaient faits avec la même durée. En fait, il n'y a pas de difficultés à introduire dans le modèle des mesures obtenues avec des temps de comptage différents.

Il suffit :

- de ramener tous les points à une échelle de temps commune (iph, par exemple),

- de pondérer chaque mesure par l'inverse de la variance qui lui est associée.

Ainsi, en fin de décroissance en particulier, il est possible d'améliorer la précision des mesures, en augmentant le temps de comptage, tout en augmentant le poids de cellesci dans l'ajustement. Ceci permet d'ajuster l'estimation des paramètres du modèle sur des valeurs de comptage qui sont, en partie, affranchies des problèmes d'instabilité électronique des échelles de mesure (de courtes périodes). 


\section{Discussion de la méthode}

Une procédure de décision, basée sur le test de STUDENT doit nous permettre de choisir l'une des 4 hypothèses possibles.

A savoir :

a) L'échantillon contient les 2 isotopes

$$
\text { Modèle 1: } c\left(t_{1}\right)=c_{1} x_{1 i}+c_{2} x_{2 i}+c_{B}
$$

b) L'échantillon contient le $1^{\text {er }}$ isotope mais pas le second (en fait, on devrait dire que l'échantillon ne contient pas une quantité suffisante du $2^{\mathrm{e}}$ isotope pour qu'il soit mis en évidence par la méthode).

$$
\text { Modèle 2: } c\left(t_{j}\right)=c_{1} x_{1 i}+c_{B}
$$

c) L'échantillon contient le $2^{\mathrm{e}}$ isotope mais non le $1^{\text {er }}$ (en fait on devrait dire que l'échantillon ne contient pas une quantité suffisante du $1^{\text {er }}$ isotope pour qu'il soit mis en évidence par la méthode).

Modèle 3: $c\left(t_{j}\right)=c_{2} x_{2 i}+c_{B}$

d) L'échantillon ne contient aucun des 2 isotopes.

Modèle $4: c\left(t_{j}\right)=c_{B}$.

Dans certains cas, peu fréquents, on peut obtenir par régression des coefficients $\hat{c}_{1}$ ou $\hat{c}_{2}$ négatifs. Il faut alors décider d'attribuer la valeur 0 à l'un ou à l'autre de ces coefficients, c'est-à-dire changer l'hypothèse du modèle.

Dans des essais avec du matériel végétal, le bruit de fond est toujours hautement significatif. Dans tous les exemples traités, le test global d'ajustement (test de FISHERSNEDECOR) permet de rejeter l'hypothèse nulle $c_{1}=c_{2}=c_{B}$ $=0 ;$ et quel que soit le modèle, le test de STUDENT associé au paramètre $C_{B}$ est significatif $\left(\hat{c}_{B} \neq 0\right)$.

Pour le choix entre les 4 modèles, les règles de décision suivantes ont été retenues. Au total 6 cas sont possibles :

On essaie le modèle 1 :

- Cas 1: si les valeurs $t$ de STUDENT associés aux paramètres $c_{1}$ et $c_{2}$ sont significatives au seuil de $\alpha=0,05$, il est retenu : les 2 isotopes sont présents.

- Cas 2: si une seule des valeurs $t$ de StUdent est significative, on retiendra le modèle correspondant, à condition que le $t$ associé à l'essai de ce modèle (2 ou 3 ) soit significatif : un seul des isotopes est présent.

Notons que ce cas est un peu délicat, car il est possible que les essais des modèles 2 et 3 conduisent à des valeurs $t$ de STUDENT significatives. Ce problème peut être dû à une corrélation trop forte entre les variables $\mathrm{x}_{1 \mathrm{i}}$ et $\mathrm{x}_{2 \mathrm{i}}$, bien que, sur le plan physique, elles ne soient pas linéairement dépendantes.

Si les 2 valeurs de $t$ de STUDENT ne sont pas significatives, on essaie les modèles 2 et 3 :

- Cas 3: le STUdent associé à $c_{1}$ (modèle 2) et celui associé à $c_{2}$ (modèle 3 ) ne sont pas significatifs. On retient le modèle 4 : l'échantillon ne contient aucun des 2 isotopes.

- Cas 4: le STUDENT associé à $c_{1}$ est significatif, celui associé à $c_{2}$ ne l'est pas. On retient le modèle 2 : présence de l'isotope 1 .

- Cas 5 : le STUDENT associé à $\mathrm{c}_{1}$ n'est pas significatif, celui associé à $c_{2}$ l'est. On retient le modèle 3 : présence de l'isotope 2.

- Cas 6 : les STUDENT associés à $c_{1}$ et $c_{2}$ sont significatifs. C'est le cas le plus délicat. On sait que l'échantillon est radioactif, mais on ne peut statuer sur la présence de l'un et/ou l'autre des 2 isotopes. C'est la marque d'une corrélation nettement trop forte entre $x_{1 i}$ et $x_{2 i}$. Il est possible de pallier cela en augmentant le nombre de mesures ou leur durée.

D'après ce qui a été développé précédemment, la qualité d'une décision dépend, en partie, du niveau de précision avec lequel les derniers comptages (fin de décroissance) ont été obtenus. Cette précision résulte d'un compromis entre des considérations pratiques (nombre d'échantillons devant être analysés) et d'autres, plus théoriques (rapport entre le temps de comptage et la plus faible des deux périodes $T_{1}$ ou $T_{2}$ ).

Il faut bien noter que la procédure de décision décrite est sans doute critiquable, mais la détermination d'une procédure optimale, compte tenu des contraintes de positivité, est certainement délicate et n'a pas été entreprise.

L'examen des courbes de décroissance facilite également l'interprétation et permet dans une certaine mesure de justifier les règles de décision adoptées.

\section{Estimation du bruit de fond des échantillons par dosage chimique du potassium foliaire}

En expérimentation, on compare généralement des individus « traités » (ici des arbres) à des individus « témoins » non traités. Le bruit de fond propre d'un échantillon inclut toutes les sources possibles de radioactivité (naturelles ou artificielles), ainsi que le bruit de l'installation; il est bien évident, mais il peut y avoir des exceptions, que le ${ }^{40} \mathrm{~K}$ est responsable de la majeure partie du comptage parasite (rapports mensuels d'activité du SCPRI). Mais il ne peut ne représenter parfois que 50 p. 100 de l'activité globale (SENS et al., 1978).

La méthode proposée au § II C permet d'estimer le bruit de fond propre de chaque échantillon $c_{B}$. Aussi nous avons mesuré par voie chimique le taux de potassium foliaire KI ( $\mathrm{K} \mathrm{mg}-\mathrm{g}^{-1}$ de poudre de feuilles séchées), après minéralisation par calcination suivie d'une reprise chlorhydrique des cendres (PINTA, 1970) afin de rechercher une relation du type :

$$
\hat{\mathrm{c}}_{\mathrm{B}}=\mathrm{aKI}+\mathrm{b},
$$

$\hat{\mathrm{C}}_{\mathrm{B}}$ étant exprimé en iph, $\mathrm{KI}$ en mg.g $\mathrm{g}^{-1}$ et b étant l'estimation du bruit de fond propre de l'appareil de mesure, coupelle et porte-coupelle compris, augmenté des contaminations éventuelles. Celles-ci devraient être très faibles, si l'on tient compte du niveau actuel des retombées radioactives, et des autres contaminants naturels (rapports SCPRI).

Les valeurs $\hat{c}_{B}$ sont estimées à partir des modèles 1,2 ou 3 si l'échantillon est radioactif. Sinon, on prend la moyenne des comptages, pondérés pour tenir compte de leur durée et de leur variance (on utilise 4 fois la moyenne horaire des comptages effectués pendant $4 \mathrm{~h}$ ).

La relation précédente est alors comparée à la courbe d'étalonnage du potassium propre à l'appareil utilisé :

$$
\mathrm{c}_{\mathrm{BK}}=\mathrm{a}^{\prime} \mathrm{K}+\mathrm{b}^{\prime} \text {, }
$$

pour laquelle on devrait avoir a' peu différent de a. Elle est obtenue par le comptage d'échantillons de poudre de feuilles auxquels on a ajouté des quantités croissantes de $\mathrm{KCl}$ (méthode des ajouts dosés). Notons que b' est nécessairement différent de b puisqu'il comprend en outre la propre teneur en potassium de la poudre végétale utilisée.

Par ailleurs, pour tester le mode d'échantillonnage des feuilles et la notion même de " témoins ", nous avons mesuré les teneurs en potassium chimique KII, sur des feuilles prélevées avant le début des injections de radioéléments dans le sol, suivant le même protocole (tabl. 4 et fig. 2). 


\section{RÉSULTATS ET INTERPRÉTATIONS}

Les valeurs des premiers comptages effectués $\left(t_{1}=0\right)$ pour l'ensemble des arbres traités ont été reportées sur le tableau 1. Pour les témoins non traités, on obtient un comptage moyen de l'ordre de $1000 \mathrm{impulsions} / \mathrm{h}$ avec une variabilité assez importante liée essentiellement au ${ }^{40} \mathrm{~K}$, comme on le verra par la suite. Le mode d'analyse des résultats qui consisterait uniquement à comparer statistiquement la $1^{\text {re }}$ séquence de comptage des échantillons traités à un bruit de fond moyen de l'ordre de $1000 \mathrm{iph}$, conduirait en première analyse, à ne retenir comme certainement radioactifs que les arbres $\mathrm{II}_{1}, \mathrm{III}_{3}$, et $\mathrm{III}_{5}$. Comme on le verra, il y a en fait 11 échantillons radioactifs, le bruit de fond réel étant différent pour chaque échantillon prélevé (cf. paragraphe suivant).

\section{TABLEAU}

Résultats des premiers comptages (iph)

Results of the first counts (cph)

\begin{tabular}{crrrr}
\hline $\begin{array}{c}\text { Rang } \\
\text { de l'arbre }\end{array}$ & I & II & III & IV \\
\hline $\begin{array}{c}\text { Numéro sur } \\
\text { la ligne }\end{array}$ & & & & \\
\hline 1 & 966 & 1.303 & 1.081 & 1.168 \\
2 & 783 & 947 & 968 & 1.003 \\
3 & 1.026 & 1.004 & 3.083 & 1.081 \\
4 & 1.003 & 1.066 & 1.098 & 992 \\
5 & 957 & 960 & 1.520 & 850 \\
6 & 975 & 1.099 & 998 & 787 \\
7 & 919 & 1.023 & 991 & 864 \\
8 & 998 & 1.066 & 891 & 1.095 \\
9 & 944 & Pas d'arbres & 1.060 \\
10 & 1.020 & \multicolumn{5}{c}{ traités } \\
\hline \hline
\end{tabular}

\section{A. Analyse du bruit de fond}

\section{Relation entre bruit de fond tet teneur en potassium foliaire}

Les valeurs des estimations $\hat{c}_{1}, \hat{c}_{2}$ et $\hat{c}_{\mathrm{B}}$ (déterminées comme il est dit au $\S$ II C) associées respectivement au phosphore, au soufre et au bruit de flond global, sont reportées sur le tableau 2 selon le modèle retenu pour l'échantillon correspondant. On y trouvera également les écart-types associés à chaque estimation. Dans 5 cas notés par un point d'interrogation, le choix entre les différents modèles n'a pu être fait.

Pour avoir une estimation de l'amplitude des variations des rendements de comptage, nous avons mesuré, au début de chaque série de comptage, le temps nécessaire à un étalon de Strontium 90 pour atteindre la valeur de $10^{5}$ impulsions. Le temps moyen est de $22,52 \mathrm{mn}$ et le coefficient de variation (rapport écart-type sur moyenne) est de 1 p. 100. La probabilité de variation de rendement durant les comptages, c'est-à-dire entre chaque passage standard, est du même ordre de grandeur.

L'examen du tableau 2 montre que l'on a pu détecter des contaminations de l'ordre de $100 \mathrm{iph}$. On observe même, pour l'arbre $\mathrm{IV}_{7}$, une contamination significative due au ${ }^{32} p$ de 54 iph pour un comptage total (contamination + bruit de fond) de $902 \mathrm{iph}$. La méthode utilisée semble donc assez sensible.

La relation entre le bruit de fond estimé $\hat{c}_{B}$ et la teneur en potassium des échantillons de feuilles est mise en évidence sur la figure 1 . On n'y a noté que les 31 points pour lesquels le choix entre les 4 modèles a été possible. On obtient par régression :

$$
\hat{\mathrm{c}}_{\mathrm{B}}=47,6 \mathrm{KI}+484 \quad \mathrm{r}^{2}=0,83 \text {. }
$$

On peut déjà constater que l'ordonnée à l'origine a une valeur compatible avec celle donnée par le constructeur pour la seule échelle de mesure.

Il est intéressant de comparer la valeur de la pente de cette droite avec celle obtenue en établissant la courbe d'étalonnage du ${ }^{40} \mathrm{~K}$ propre à l'appareil utilisé (cf. tabl. 3). Cette

TABLEAU 2

Modèles retenus et estimation des bruits de fond correspondants

Models selected for study and fitted background values

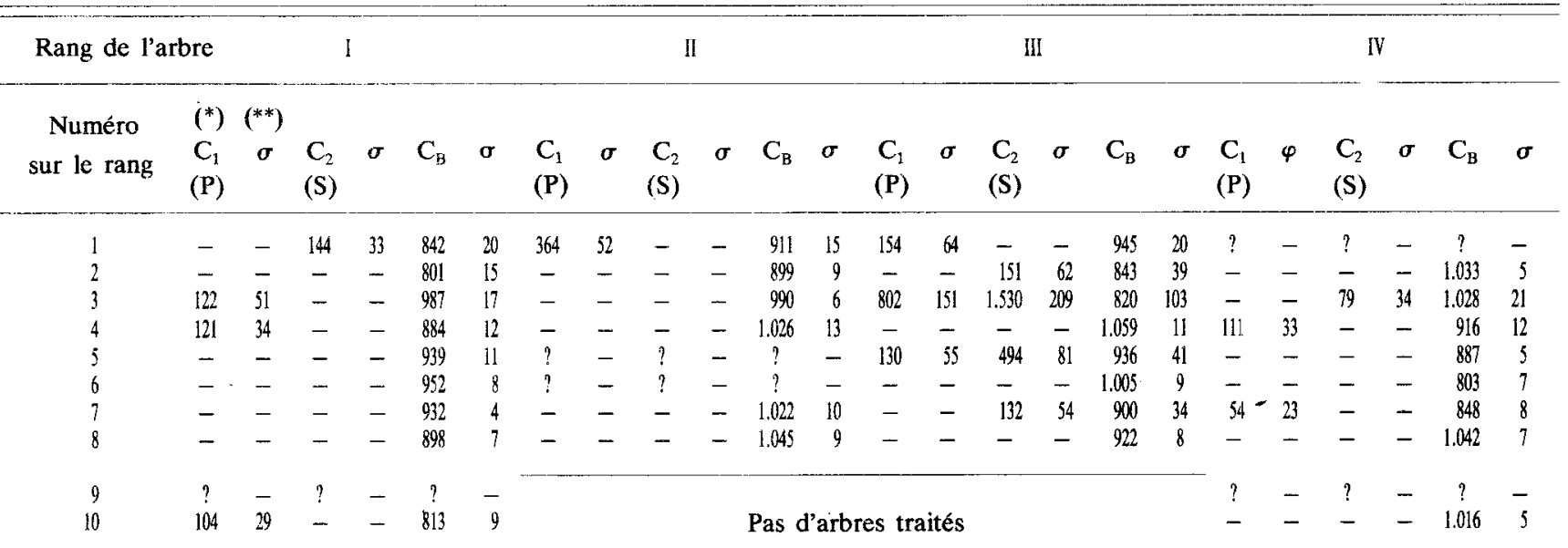




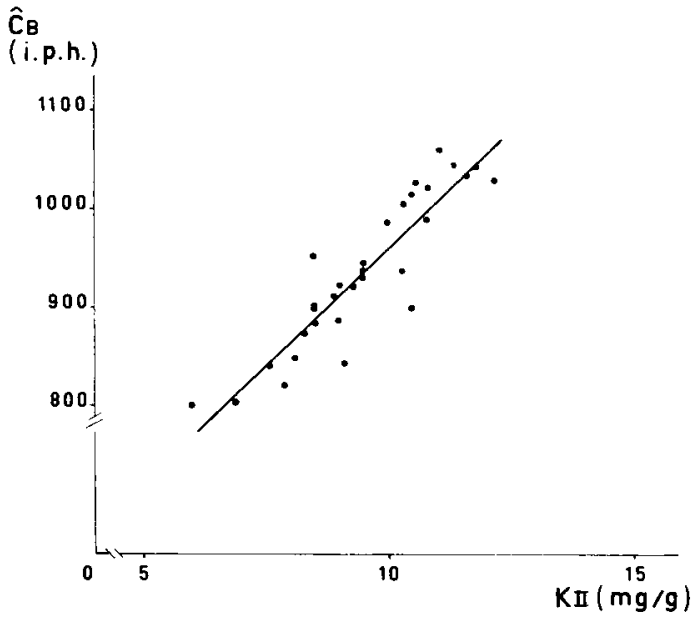

Figure 1

Relation entre le bruit de fond estimé et le taux de potassium mesuré.

Fitted background plotted versus measured potassium content.

courbe est une droite d'équation (après ajustement par les moindres carrés :

$$
\mathrm{c}_{\mathrm{BK}}=40,8 \mathrm{~K}+964 \quad \mathrm{r}^{2}=0,98 .
$$

Les valeurs des 2 pentes sont suffisamment voisines (en fait, elle ne sont pas significativement différentes au seuil $\alpha=0,05$ avec $t_{\text {obs }}=1,65$ ) pour qu'on accepte leur égalité. Il est cependant probable qu'une partie de la différence observée soit due à l'effet de radioisotopes autres que le potassium 40 . Cette comparaison permet cependant de conclure que c'est bien au ${ }^{40} \mathrm{~K}$ qu'est due la majeure partie du bruit de fond végétal, bien que cela ne soit pas notre propos.

Le fait que les 2 pentes aient une valeur assez voisine permet de poser que le mode d'estimation des bruits de fond (c.à.d. le choix entre les 4 modèles) conduit à des résultats cohérents quant à leurs valeurs. Ainsi, on peut penser que la procédure de tests utilisés permet de distinguer les échantillons radioactifs de ceux qui ne le sont pas.

Un autre but de ce travail était de permettre la discrimination des deux isotopes utilisés. D'après ce qui a été dit au paragraphe 1 , il apparaît :

- qu'elle se fait sans doute dans de bonnes conditions pour les cas $1,4,5$ ( $\$$ II C),

- que pour le cas 2, par contre, il est parfois nécessaire d'obtenir un complément de mesures. Ceci est à apprécier par l'utilisateur, en fonction, par exemple, de l'intervalle de confiance de l'estimateur jugé significativement différent de 0 , ou des résultats des 2 régressions simples.

\section{Variabilité du taux de potassium foliaire}

On vient de montrer que $1 \mathrm{mg} . \mathrm{g}^{-1}$ de potassium induit environ $50 \mathrm{iph}$ (ceci correspond à un rendement dans les conditions de mesures utilisées de l'ordre de 50 p. 100 , alors que les niveaux de contamination observés sont compris le plus souvent entre 50 et 150 iph (tabl. 3). Aussi, a-t-on voulu juger de la représentativité d'un échantillon de feuilles quant à sa teneur en potassium si l'on veut comparer, d'une part, 2 échantillons d'un même arbre et, d'autre part, différents arbres.

Sur le tableau 4 sont notées les valeurs des teneurs chimiques en potassium foliaire résultant de 2 échantillonnages effectués au cours de la journée, notés KI pour le $1^{\text {er }}$ et KII pour le $2^{\mathrm{e}}$.

Pour donner un exemple, la moyenne générale pour le $1^{\text {er }}$ échantillonnage vaut $\mathrm{KI}=9,6$, avec un écart-type de 1,8 , alors que, pour le $2^{e}$, on a KII $=9,5$ avec un écarttype de 1,5 .

Pour tester si le mode d'échantillonnage est reproductible, on peut calculer la valeur de la variable de STUDENT associée à la différence.

\section{$\mathrm{d}=\mathrm{KI}$ - KII (test par paire).}

On en conclut que $\mathrm{d}$ n'est pas significativement différent de 0 au seuil $\alpha=0,05\left(\mathrm{t}_{\mathrm{obs}}=0,25\right)$ et donc que les 2 échantillonnages ne sont pas statistiquement différents. Cependant, l'écart-type associé à $\mathrm{d}$ vaut 0,8 . Donc des différences, pour un même arbre, entre 2 échantillonnages, de teneur en potassium de l'ordre de 1 à $1,5 \mathrm{mg} . \mathrm{g}^{-1}$ ne sont pas à écarter. Rappelons que cela correspond à des variations du bruit de fond de l'ordre de 50 à 75 iph.

L'étude du tableau 4 montre, par ailleurs, que la dispersion due aux arbres est, pour une bonne part, liée à leurs rangs. On observe sans doute là un effet de compétition entre les pommiers eux-mêmes et entre le rang I et la haie de cyprès. Cependant, même au niveau des rangs médians II et III, on note une variation du taux de potassium foliaire importante. Elle peut atteindre presque $3 \mathrm{mg} \cdot \mathrm{g}^{-1}$ soit 150 iph en terme de variation de bruit de fond.

La dispersion des résultats est montrée sur la figure 2 où sont reportés les 36 couples (KI, KII). Cette dispersion est à apprécier, d'une part, par rapport à la droite passant par la $1^{\mathrm{re}}$ bissectrice (dispersion liée au mode d'échantillonnage) et, d'autre part, le long de cette droite (dispersion liée aux arbres).

Ce travail rappelle qu'il est nécessaire pour des faibles niveaux de radioactivité d'estimer le bruit de fond propre des échantillons. Notons que la méthode proposée permet de prendre en compte le cas d'échantillons dont le bruit de

TABLEAU 3

Etalonnage de l'échelle de mesure pour le potassium

Potassium apparatus calibration

\begin{tabular}{|c|c|c|c|c|c|c|c|c|c|c|c|}
\hline $\begin{array}{l}\text { Concentration } \\
\text { en } K \text { de la poudre } \\
\text { de feuille (mg. } \mathrm{g}^{-1} \text { ) }\end{array}$ & 0 & 0 & 3 & 3 & 6 & 9 & 9 & 12 & 12 & 15 & 15 \\
\hline $\begin{array}{l}\mathrm{C}_{\mathrm{BK}}{ }^{*} \\
\text { (iph) }\end{array}$ & 963 & 957 & 1.080 & 1.075 & 1.214 & 1.357 & 1.347 & 1.456 & 1.479 & 1.540 & 1.571 \\
\hline
\end{tabular}

$\left(^{*}\right)$ Les comptages ont été faits pendant une durée de $4 \mathrm{~h}$ et ramenés à l'échelle iph

(*) Samples counted for $4 \mathrm{~h}$ and results then expressed as $\mathrm{c} / \mathrm{h}$. 
TABLEAU 4

Teneurs en potassium des premiers et seconds échantillons de feuilles ( $m g . \mathrm{g}^{-1}$ ) Potassium contents of the first and second leaf samples (mg. $\mathrm{g}^{-1}$ )

\begin{tabular}{|c|c|c|c|c|c|c|c|c|}
\hline $\begin{array}{c}\begin{array}{c}\text { Rang } \\
\text { de l'arbre }\end{array} \\
\begin{array}{c}\text { Numéro sur } \\
\text { la ligne }\end{array}\end{array}$ & \multicolumn{2}{|c|}{ I } & \multicolumn{2}{|c|}{ II } & \multicolumn{2}{|c|}{ III } & \multicolumn{2}{|c|}{ IV } \\
\hline 1 & 6.8 & 7.6 & 9.1 & 8.9 & 9.0 & 9.5 & 15.0 & 13.6 \\
\hline 3 & 9.6 & 10.0 & 11.0 & 10.8 & 9.2 & 7.9 & 11.8 & 12.2 \\
\hline 4 & 8.4 & 8.5 & 10.2 & 10.6 & 10.2 & 11.1 & 9.8 & 9.0 \\
\hline 5 & 9.5 & 9.5 & 9.0 & 8.5 & 11.8 & 10.3 & 8.4 & 9.0 \\
\hline 6 & 7.6 & 8.5 & 10.6 & 9.4 & 10.5 & 10.3 & 7.5 & 6.9 \\
\hline 7 & 8.8 & 9.5 & 11.0 & 10.8 & 10.9 & 10.5 & 9.0 & 8.1 \\
\hline 8 & 8.0 & 8.5 & 10.4 & 11.4 & 9.6 & 9.3 & 10.9 & 11.8 \\
\hline 9 & 6.8 & 7.6 & \multicolumn{3}{|c|}{ Pas d'arbres traités } & & 10.5 & 10.0 \\
\hline 10 & 7.1 & 8.3 & - & - & - & - & 11.1 & 10.5 \\
\hline Ecart-type & 1.2 & 1.2 & 0.8 & 1.2 & 1.1 & 1.0 & 2.2 & 2.1 \\
\hline $\begin{array}{l}\left({ }^{*}\right) \mathrm{K}_{\mathrm{I}} \text { est la teneur en } \\
\left({ }^{* *}\right) \mathrm{K}_{\mathrm{II}} \text { est la teneur } \\
\left({ }^{*}\right) \mathrm{K}_{\mathrm{I}} \text { is the potassiur } \\
\left({ }^{* *}\right) \mathrm{K}_{\mathrm{II}} \text { is the potassi }\end{array}$ & $\begin{array}{l}\text { du } 1^{\text {er éc }} \text { é } \\
\text { he leav }\end{array}$ & $\begin{array}{l}\text { lon de } \mathrm{f} \\
\text { llon de } \\
\text { npled in }\end{array}$ & $\begin{array}{l}\text { préle } \\
\text { s prél } \\
\text { ornir }\end{array}$ & $\begin{array}{l}\text { latin } \\
\text { cours }\end{array}$ & urné & & & \\
\hline
\end{tabular}

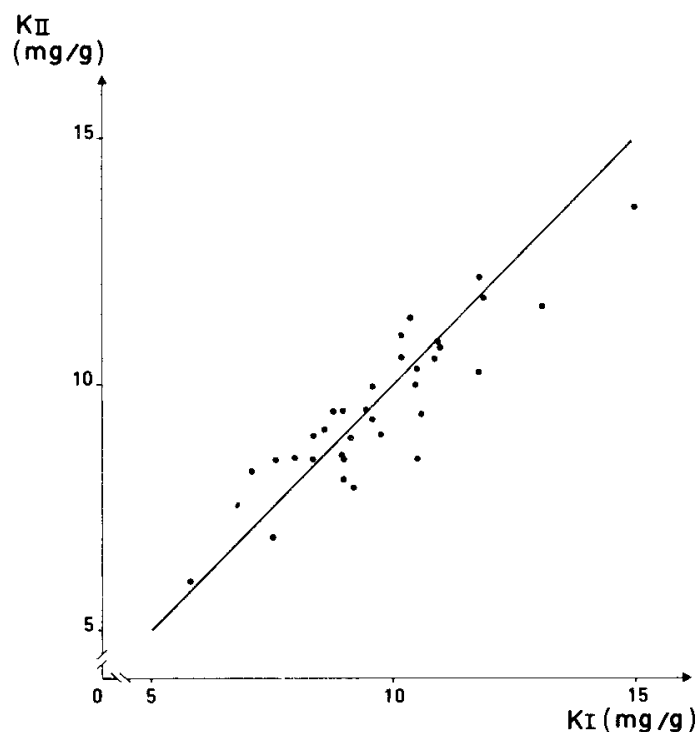

Figure 2

Dispersion des couples $\left.\left(K_{I}\left({ }^{*}\right), K_{I I}{ }^{* *}\right)\right)$ par rapport à la Ire bissectrice. $\left.{ }^{*}\right) K_{I}$ est la teneur en potassium du $1^{\text {er }}$ échantillon de feuilles prélevé le matin

$\left(^{* *}\right) K_{I I}$ est la teneur en potassium du $2^{e}$ échantillon de feuilles prélevé au cours de la journée.

Scatter of the $K_{I}\left(^{*}\right), K_{I I}\left(^{* *}\right)$ couples around the first bisecting line. $\left.{ }^{*}\right) K_{I}$ is the potassium content of the leaves sampled in the morning. (**) $K_{I I}$ is the potassium content of the leaves sampled during the day.

fond n'est pas lié uniquement, ou principalement, au potassium, ce qui a été parfois montré (SENS et al. 1978).

Par ailleurs, il faut souligner que, dans le cas de l'utilisation d'un seul radioisotope, la procédure statistique de décision est considérablement simplifiée. En effet, l'origine de la radioactivité, si la valeur du test de STUDENT cor- respondant est significative, ne pose aucune ambiguïté. La méthode paraît alors particulièrement bien adaptée au cas de végétaux faiblement contaminés et fortement variables quant à leurs états initiaux ; ce qui est généralement le cas des arbres fruitiers. Les résultats des tableaux 1 et 4 le montrent clairement.

\section{B. Examen des courbes de décroissance}

Nous avons déjà remarqué que les tests utilisés pour choisir entre les 4 modèles possibles $(P+S, P, S, O)$ n'étaient pas optimaux. Aussi est-il nécessaire de confronter leurs réalisations aux courbes expérimentales, en prenant garde toutefois au caractère subjectif attaché à ce type d'analyse.

Dans la figure 3, on a représenté 6 graphiques qui correspondent aux 6 modalités de décision décrites au paragraphe II C 3. Nous avons tenté de faire une comparaison graphique entre le modèle retenu (courbes en trait plein) et le(s) modèle(s) rejeté(s) qui s'y opposai(en)t le plus directement (courbe en tirets). Ainsi :

- si la décision a été de retenir le modèle $1(P+S)$, on lui a opposé les modèles 2 et 3 (figure $3 a$ ).

- si la décision a été de retenir le modèle $2(\mathrm{P})$, on lui a opposé le modèle 3 (S) (figure $3 b$, d) et vice-versa (figure 3e),

- si la décision a été de retenir le modèle $4(\mathrm{O})$, on lui a opposé les modèles 2 et 3 (figure 3c)

- s'il y a eu indécision, on a représenté les modèles 2 et 3 (figure $3 f$ ).

Il apparaît à l'examen de la figure 3 que le modèle retenu est généralement celui qui :

- d'une part, s'ajuste le mieux aux points expérimentaux ; c'est par construction celui qui a la variance résiduelle la plus faible pour les graphiques $a, b, d$ et $e$.

- d'autre part, paraît respecter le mieux l'orientation (pente de la courbe) que dessinent les points à variance 4 fois 

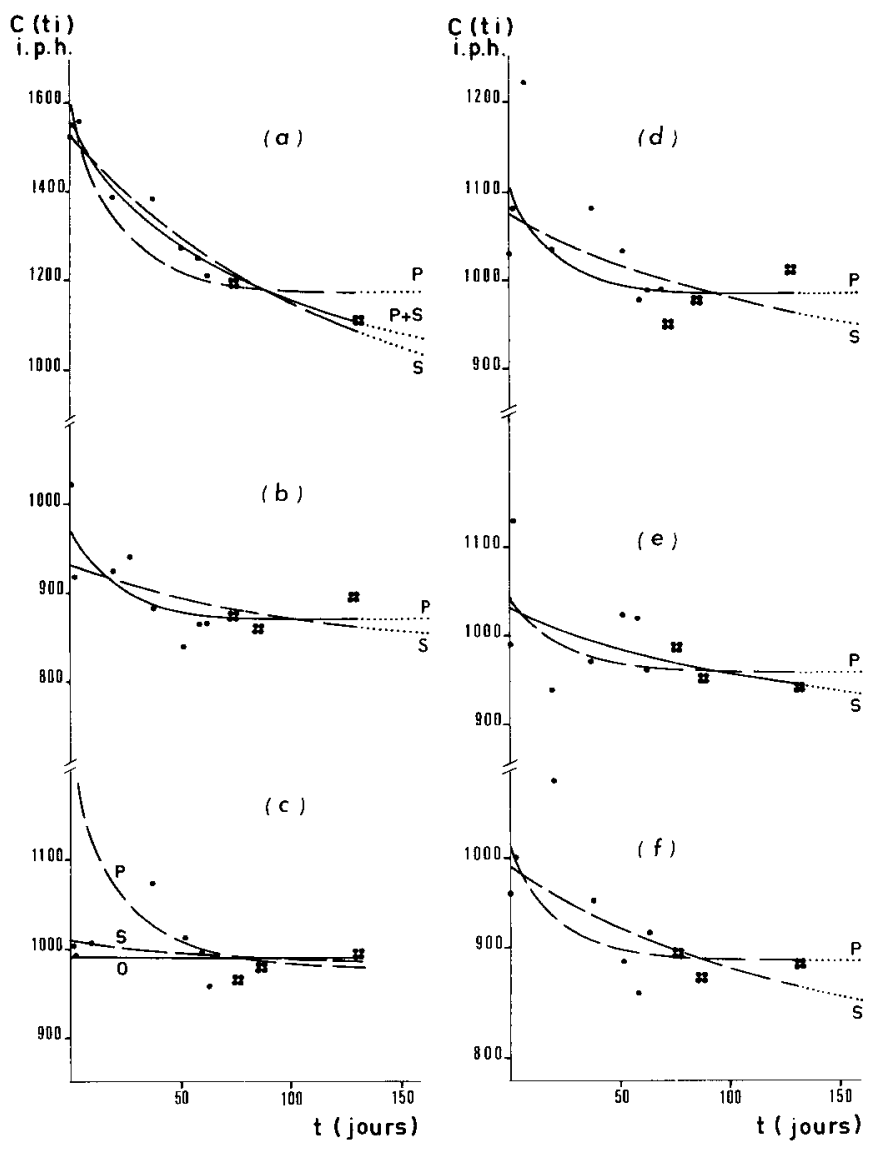

Figure 3

Courbes de décroissance : exemples d'application des procédures de décision

Decrease curves: examples of the use of decision rules

(a) Cas $1(P+S)$ arbre: $I I_{5}$; Rule $1(P+S)$ : tree $I I_{5}$

(b) Cas $2(P)$ : arbre $I_{10}$; Rule $2(P)$ : tree $I_{10}$

(c) Cas $3(\mathrm{O})$ : arbre $\mathrm{II}_{3}$; Rule $3(\mathrm{O})$ : tree $\mathrm{I}_{3}$

(d) Cas $4(P)$ : arbre $I_{3}$; Rule $4(P)$ : tree $I_{3}$

(e) Cas 5 (S): arbre III $\mathrm{I}_{7}$; Rule 5 (S): tree $\mathrm{III}_{7}$

(f) Cas 6 (?): arbre $I I_{5}$; Rule 6 (?): tree $I_{5}$.

plus faible, marqués par 4 points en carré sur les graphiques, car résultant d'un comptage de $4 \mathrm{~h}$ (cf. la partie extrapolée des courbes, en trait pointillé).

A cet égard, l'examen de la figure $3 f$ est intéressant. L'indécision semble se marquer par une quasi-équivalence graphique, subjective certes, des modèles 2 et 3 .

Il faut noter par ailleurs que, dans la majorité des cas, la différence entre les comptages expérimentaux et les comptages estimés (découlant de l'utilisation du modèle), soit l'erreur $\mathrm{e}_{\mathrm{i}}$, est du même ordre de grandeur que celle qui était prévue $\left(\mathrm{e}_{\mathrm{i}} \leqslant 2 . \mathrm{C}\left(\mathrm{t}_{\mathrm{i}}{ }^{1 / 2}\right)\right.$, ce qui conforte les hypothèses que nous avions faites sur la nature des erreurs.

\section{CONCLUSIONS}

La mesure du taux de comptage dû au ${ }^{32} \mathrm{P}$ au moyen d'un compteur à flux gazeux ne pose, en principe, aucune diffi- culté pour des échantillons végétaux séchés et broyés. Par contre, la mesure de ${ }^{35} \mathrm{~S}\left(\mathrm{E}_{\max }=0,167 \mathrm{Mev}\right)$ ne peut être réalisée qu'avec des activités en général plus élevées que pour ${ }^{32} \mathbf{P}$, compte tenu des rendements des ensembles de comptage à flux gazeux pour une telle énergie.

Soulignons le fait que la méthode proposée ne comporte pas le dosage chimique du potassium qui n'a été effectué ici que pour analyser la variabilité du bruit de fond global, lequel est estimé avec, en définitive, la précision de l'ajustement des données au modèle retenu.

Une amélioration intéressante serait d'éliminer chimiquement le potassium de l'échantillon afin de ramener le bruit de fond au niveau du mouvement propre de l'enceinte de mesure, soit de l'ordre de $400 \mathrm{iph}$, ce qui augmenterait la sensibilité pour ${ }^{32} \mathrm{P}$ et ${ }^{35} \mathrm{~S}$ et permettrait une décision d'absence ou de présence pour quelques échantillons supplémentaires. Dans le même ordre d'idées, on pourrait améliorer les prises de décision en séparant chimiquement phospore et soufre que l'on compterait soit par un équipement à flux gazeux, soit en scintillation liquide (en comptage Cerenkov ou avec scintillateur). Par rapport au bruit de fond des installations courantes, pour ce dernier type de technique, il serait cependant encore nécessaire de procéder à des comptages relativement longs. De plus, par suite de l'évolution des suspensions utilisées, tous les échantillons ne seraient alors plus comparables quant au degré de signification du résultat obtenu.

Pour conclure, on peut dire que la méthode proposée offre surtout l'inconvénient de sa durée globale, qui est cependant fonction des périodes des radioisotopes utilisés. Dans le cas d'un double marquage, on peut estimer, par expérience, cette durée à 1,5 fois la demi-vie de l'isotope ayant la période la plus longue. Dans le cas d'un marquage avec un seul radioisotope, la durée à utiliser est plus faible. Ainsi peut-on penser qu'avec un marquage au ${ }^{32} \mathrm{P}$, environ 2 semaines suffiraient pour obtenir les résultats.

Cependant, cette méthode est très facile à mettre en œuvre, aussi bien pour la préparation des échantillons que pour les programmes de calcul utilisés. Ainsi, si on travaille avec un seul radioisotope, il est possible de les effectuer avec une calculatrice de bureau programmable. Un autre avantage, sans doute plus décisif, est qu'elle permet d'accéder, sans manipulation, à la prise en compte du bruit de fond propre de l'échantillon mesuré. Ceci permet d'éviter la pratique du double échantillonnage (témoin/essai) qui n'est pas sans poser de problèmes du fait de la variabilité présentée par des échantillons contaminés ou non contaminés dans l'ensemble d'un essai.

Il est bien évident que l'utilisation d'appareils de série, non améliorés quant à leur mouvement propre, introduit une limitation de sensibilité qui diminue le nombre de cas pour lesquels on peut prendre une décision de contamination. Pour relier cette contamination, donc une absorption racinaire, aux facteurs physiques du milieu que l'on veut tester (НАвІв, 1981), il importe donc de concevoir le dispositif expérimental de manière à avoir suffisamment d'individus pour lesquels la décision prise sera significative. 


\section{RÉFÉRENCES BIBLIOGRAPHIQUES}

Bachacou J., Masson J..P., Millier C., 1981. Manuel de la programmatheque statistique. Amance 81, INRA-CNRF Nancy, Département de Biométrie, 516 p.

Broeshart H., Nethsing D.A., 1972. Studies on the pattern of root activity of tree crops using isotopes techniques. IAEA, Isotopes and radiation in soil-plants relationships including Forestry, 465-479.

Habib R., 1981. Etude pour les végétaux pérennes (pommiers) soumis à une irrigation localisée, des conditions d'absorption racinaire du phosphore. Thèse U.S.T.L. Montpellier, $70 \mathrm{p}$.

IAEA, 1967. Radioactive dating and methods of low-level counting. Monaco Symposium, 2-10 March 1967, 744 p.

IAEA, 1981. Methods of low-level counting and spectrometry. Berlin (West) Symposium, 6-10 April 1981, 556 p.
Pinta M., 1970. Méthodes de référence pour la détermination des éléments minéraux dans les végétaux in "Le contrôle de la fertilisation des plantes cultivées $»$. II. Coloquio Europeo y Mediterraneo, Sevilla, 103-122.

Quittner P., 1973. Gamma ray spectroscopy. Adam Hilger LTD, London, $111 \mathrm{p}$.

Rapports d'activité du SCPRI-INSERM, Le Vésinet.

Sens J.-C., Pape A., Dephin J., Marocke R., Mettauer H., Wagner R., 1978. Définition de l'état actuel de la radioactivité, in Contribution à l'étude écologique de la région de Fessenheim. II Annexe. Imprimé au Centre de Recherches Nucléaires de Strasbourg, décembre 1978, $172 \mathrm{p}$. 